\title{
More Amicable Numbers
}

\section{By Paul Bratley and John McKay}

A recent paper [1] listed all pairs of amicable numbers below $10^{6}$. The following extension contains all amicable pairs with the lesser number between $10^{6}$ and 107. Pairs marked with an asterisk are not found in the lists given by Escott [2], Poulet [3], and Garcia [4]. However, our referee has kindly pointed out that 22 of these pairs appear in a paper by Lee [5] which was in the process of publication. The 16 pairs marked $\dagger$ appear to be new, and complete the list of 108 pairs less than $10^{7}$.

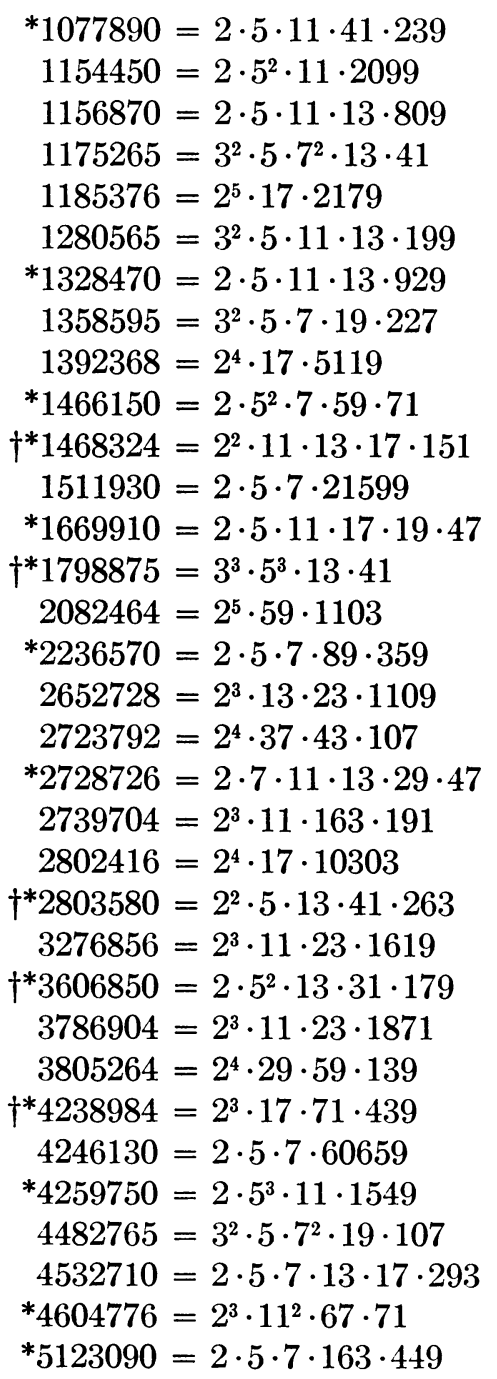

$$
\begin{aligned}
& 1099390=2 \cdot 5 \cdot 17 \cdot 29 \cdot 223 \\
& 1189150=2 \cdot 5^{2} \cdot 17 \cdot 1399 \\
& 1292570=2 \cdot 5 \cdot 19 \cdot 6803 \\
& 1438983=3^{2} \cdot 7^{2} \cdot 13 \cdot 251 \\
& 1286744=2^{3} \cdot 41 \cdot 3923 \\
& 1340235=3^{2} \cdot 5 \cdot 13 \cdot 29 \cdot 79 \\
& 1483850=2 \cdot 5^{2} \cdot 59 \cdot 503 \\
& 1486845=3^{2} \cdot 5 \cdot 19 \cdot 37 \cdot 47 \\
& 1464592=2^{4} \cdot 239 \cdot 383 \\
& 1747930=2 \cdot 5 \cdot 47 \cdot 3719 \\
& 1749212=2^{2} \cdot 37 \cdot 53 \cdot 223 \\
& 1598470=2 \cdot 5 \cdot 19 \cdot 47 \cdot 179 \\
& 2062570=2 \cdot 5 \cdot 239 \cdot 863 \\
& 1870245=3^{2} \cdot 5 \cdot 13 \cdot 23 \cdot 139 \\
& 2090656=2^{5} \cdot 79 \cdot 827 \\
& 2429030=2 \cdot 5 \cdot 23 \cdot 59 \cdot 179 \\
& 2941672=2^{3} \cdot 71 \cdot 5179 \\
& 2874064=2^{4} \cdot 263 \cdot 683 \\
& 3077354=2 \cdot 7 \cdot 19 \cdot 23 \cdot 503 \\
& 2928136=2^{3} \cdot 31 \cdot 11807 \\
& 2947216=2^{4} \cdot 167 \cdot 1103 \\
& 3716164=2^{2} \cdot 503 \cdot 1847 \\
& 3721544=2^{3} \cdot 647 \cdot 719 \\
& 3892670=2 \cdot 5 \cdot 29 \cdot 31 \cdot 433 \\
& 4300136=2^{3} \cdot 467 \cdot 1151 \\
& 4006736=2^{4} \cdot 179 \cdot 1399 \\
& 4314616=2^{3} \cdot 23 \cdot 131 \cdot 179 \\
& 4488910=2 \cdot 5 \cdot 23 \cdot 29 \cdot 673 \\
& 4445050=2 \cdot 5^{2} \cdot 19 \cdot 4679 \\
& 5120595=3^{2} \cdot 5 \cdot 19 \cdot 53 \cdot 113 \\
& 6135962=2 \cdot 7 \cdot 71 \cdot 6173 \\
& 5162744=2^{3} \cdot 113 \cdot 5711 \\
& 5504110=2 \cdot 5 \cdot 19 \cdot 59 \cdot 491 \\
& =20
\end{aligned}
$$

Received September 13, 1967. 


\begin{tabular}{|c|c|}
\hline $5147032=2^{3} \cdot 11 \cdot 23 \cdot 2543$ & $5843048=2^{3} \cdot 383 \cdot 1907$ \\
\hline$\dagger^{*} 5232010=2 \cdot 5 \cdot 7 \cdot 41 \cdot 1823$ & $5799542=2 \cdot 7^{2} \cdot 23 \cdot 31 \cdot 83$ \\
\hline${ }^{*} 5357625=3 \cdot 5^{3} \cdot 7 \cdot 13 \cdot 157$ & $5684679=3^{2} \cdot 7 \cdot 11 \cdot 13 \cdot 631$ \\
\hline$\dagger^{*} 5385310=2 \cdot 5 \cdot 7 \cdot 107 \cdot 719$ & $5812130=2 \cdot 5 \cdot 17 \cdot 179 \cdot 191$ \\
\hline${ }^{*} 5459176=2^{3} \cdot 17 \cdot 137 \cdot 293$ & $5495264=2^{5} \cdot 83 \cdot 2069$ \\
\hline$\dagger^{*} 5726072=2^{3} \cdot 11 \cdot 31 \cdot 2099$ & $6369928=2^{3} \cdot 79 \cdot 10079$ \\
\hline${ }^{*} 5730615=3^{3} \cdot 5 \cdot 11 \cdot 17 \cdot 227$ & $6088905=3^{3} \cdot 5 \cdot 23 \cdot 37 \cdot 53$ \\
\hline$\dagger^{*} 5864660=2^{2} \cdot 5 \cdot 17 \cdot 47 \cdot 367$ & $7489324=2^{2} \cdot 53 \cdot 35327$ \\
\hline${ }^{*} 6329416=2^{3} \cdot 23 \cdot 41 \cdot 839$ & $6371384=2^{3} \cdot 19 \cdot 167 \cdot 251$ \\
\hline$* 6377175=3^{2} \cdot 5^{2} \cdot 7 \cdot 4049$ & $6680025=3^{2} \cdot 5^{2} \cdot 11 \cdot 2699$ \\
\hline $6955216=2^{4} \cdot 19 \cdot 137 \cdot 167$ & $7418864=2^{4} \cdot 463679$ \\
\hline $6993610=2 \cdot 5 \cdot 13 \cdot 23 \cdot 2339$ & $7158710=2 \cdot 5 \cdot 13 \cdot 53 \cdot 1039$ \\
\hline$\dagger^{*} 7275532=2^{2} \cdot 11 \cdot 37 \cdot 41 \cdot 109$ & $7471508=2^{2} \cdot 11^{2} \cdot 43 \cdot 359$ \\
\hline$* 7288930=2 \cdot 5 \cdot 11 \cdot 23 \cdot 43 \cdot 67$ & $8221598=2 \cdot 7 \cdot 11 \cdot 197 \cdot 271$ \\
\hline$* 7489112=2^{3} \cdot 17 \cdot 53 \cdot 1039$ & $7674088=2^{3} \cdot 23 \cdot 179 \cdot 233$ \\
\hline$* 7577350=2 \cdot 5^{2} \cdot 11 \cdot 23 \cdot 599$ & $8493050=2 \cdot 5^{2} \cdot 59 \cdot 2879$ \\
\hline $7677248=2^{6} \cdot 139 \cdot 863$ & $7684672=2^{6} \cdot 167 \cdot 719$ \\
\hline $7800544=2^{5} \cdot 43 \cdot 5669$ & $7916696=2^{3} \cdot 17 \cdot 58211$ \\
\hline$* 7850512=2^{4} \cdot 37 \cdot 89 \cdot 149$ & $8052488=2^{3} \cdot 29 \cdot 61 \cdot 569$ \\
\hline${ }^{*} 8262136=2^{3} \cdot 17 \cdot 79 \cdot 769$ & $8369864=2^{3} \cdot 29 \cdot 43 \cdot 839$ \\
\hline$\dagger^{*} 8619765=3 \cdot 5 \cdot 7 \cdot 11 \cdot 17 \cdot 439$ & $9627915=3 \cdot 5 \cdot 11 \cdot 23 \cdot 43 \cdot 59$ \\
\hline$* 8666860=2^{2} \cdot 5 \cdot 23 \cdot 83 \cdot 227$ & $10638356=2^{2} \cdot 47 \cdot 71 \cdot 797$ \\
\hline $8754130=2 \cdot 5 \cdot 7 \cdot 11 \cdot 11369$ & $10893230=2 \cdot 5 \cdot 757 \cdot 1439$ \\
\hline$\dagger^{*} 8826070=2 \cdot 5 \cdot 11 \cdot 19 \cdot 41 \cdot 103$ & $10043690=2 \cdot 5 \cdot 31 \cdot 179 \cdot 181$ \\
\hline $9071685=3^{2} \cdot 5 \cdot 7 \cdot 31 \cdot 929$ & $9498555=3^{2} \cdot 5 \cdot 11 \cdot 31 \cdot 619$ \\
\hline $9199496=2^{3} \cdot 19 \cdot 29 \cdot 2087$ & $9592504=2^{3} \cdot 29 \cdot 173 \cdot 239$ \\
\hline$\dagger^{*} 9206925=3 \cdot 5^{2} \cdot 7 \cdot 13 \cdot 19 \cdot 71$ & $10791795=3 \cdot 5 \cdot 7 \cdot 79 \cdot 1301$ \\
\hline$* 9339704=2^{3} \cdot 11 \cdot 211 \cdot 503$ & $9892936=2^{3} \cdot 47 \cdot 83 \cdot 317$ \\
\hline $9363584=2^{7} \cdot 191 \cdot 383$ & $9437056=2^{7} \cdot 73727$ \\
\hline$\dagger^{*} 9478910=2 \cdot 5 \cdot 7 \cdot 19 \cdot 7127$ & $11049730=2 \cdot 5 \cdot 71 \cdot 79 \cdot 197$ \\
\hline$\dagger^{*} 9491625=3^{2} \cdot 5^{3} \cdot 11 \cdot 13 \cdot 59$ & $10950615=3^{2} \cdot 5 \cdot 13 \cdot 18719$ \\
\hline$* 9660950=2 \cdot 5^{2} \cdot 13 \cdot 89 \cdot 167$ & $10025290=2 \cdot 5 \cdot 11 \cdot 91139$ \\
\hline$\dagger^{*} 9773505=3^{2} \cdot 5 \cdot 7 \cdot 19 \cdot 23 \cdot 71$ & $11791935=3^{2} \cdot 5 \cdot 31 \cdot 79 \cdot 107$ \\
\hline
\end{tabular}

It is conjectured that all odd amicable numbers are divisible by 3 . (The apparent counterexample on p. 168 of Garcia's paper is a misprint, the common factor $2^{3}$ being missing.)

\footnotetext{
Department of Computer Science

University of Edinburgh

Edinburgh, Scotland
}

Atlas Computer Laboratory

Chilton, Didcot, England

1. J. Alanen, O. Ore, \& J. Stemple, "Systematic computations on amicable numbers," Math. Comp., v. 21, 1967, pp. 242-245.

2. E. B. EscotT, "Amicable numbers," Scripta Math., v. 12, 1946, pp. 61-72. MR 8, 135.

3. P. POULET, "43 new couples of amicable numbers," Scripta Math., v. 14, 1948, p. 77.

4. M. GARCIA, "New amicable pairs," Scripta Math., v. 23, 1957, pp. 167-171. MR 20 \#158.

5. E. J. LEE, "Amicable numbers and the bilinear diophantine equation," Math. Comp., v. 22,1968 , pp. 181-187. 